Copyright by the Acoustical Society of America. Ruckman, C. E., \& Fuller, C. R. (1995). A regression approach for simulating feedforward active noise control. Journal of the Acoustical Society of America, 97(5), 2906-2918. doi: 10.1121/1.411857

\title{
A regression approach for simulating feedforward active noise control
}

\author{
Christopher E. Ruckman \\ Structural Acoustics and Hydroacoustics Research Branch, Carderock Division, Naval Surface Warfare \\ Center, Bethesda, Maryland 20084-5000
}

\author{
Chris R. Fuller \\ Vibration and Acoustics Laboratories, Virginia Polytechnic Institute and State University, Blacksburg, \\ Virginia 24061
}

(Received 21 April 1994; revised 29 September 1994; accepted 24 November 1994)

\begin{abstract}
Regression analysis is used to examine feedforward active noise control from a statistical point of view. Since numerical techniques for simulating feedforward active noise control in the frequency domain are mathematically similar to linear least-squares regression, two regression-based numerical methods can be applied to control problems. The first uses regression diagnostics such as the $F$-test, the $t$-test, and confidence intervals to model the effects of error sensor measurement noise. The second uses collinearity diagnostics to address a form of numerical ill conditioning that can corrupt the results. The regression diagnostics allow realistic modeling of random measurement error; the collinearity diagnostics help avoid numerical difficulties that might otherwise go undetected. Numerical results are given for a structural-acoustic control problem involving a fluid-loaded cylindrical shell.
\end{abstract}

PACS numbers: $43.40 . \mathrm{Vn}$

\section{INTRODUCTION}

This article examines feedforward active noise control from a statistical viewpoint using tools developed for linear least-squares regression. The procedure for simulating feedforward active control using, for example, a finite-element model of the plant, can be conveniently simulated in the frequency domain using a formulation that is essentially identical to regression; the main difference is that the regression variables are complex-valued transfer functions rather than real-valued collections of data observations. Regression diagnostics can be used to assess the effects of error sensor measurement noise, which is analogous to observation error in a regression. Collinearity diagnostics can be used to detect and analyze ill-conditioning problems that might otherwise go unnoticed. Together, these numerical methods provide a unified numerical approach for simulating active control systems.

Consider a single-input, single-output (SISO) feedforward active control system. A primary or disturbance input $S$ injects vibrational or acoustic energy, while one or more actuators (secondary or control inputs) are used to influence system response. The disturbance propagates through the primary path $A$ to form the disturbance response (primary response) $P_{p}$. The controller operates on a detection sensor signal to produce the control input $b$, which propagates through the secondary path $X$ to form the control response $P_{c}$. The total response $P_{p}+P_{c}$ is measured by the error sensor subject to measurement noise $\epsilon$, and the error sensor output is used in determining the controller gain. Frequencydomain investigations of this type of system have been reported in a variety of contexts. ${ }^{1-5}$

The need for regression diagnostics stems from two facts. First, error sensor outputs in a real control system are contaminated with measurement noise whose properties are known only in a statistical sense. Second, in a MIMO system the error is known only at a finite number of locations (the error sensors) rather than as a continuous function in space. For these two reasons among others, numerical or analytical simulations are usually too optimistic in their predictions of system performance. To make simulations more realistic, regression diagnostics can be used to characterize the effects of measurement noise. This implicit stochastic modeling of measurement noise differentiates the statistical methods outlined below from previous works, which consider the problem from a purely deterministic viewpoint. In keeping with the tutorial nature of the discussion, we discuss only the three most basic regression diagnostics: the $F$-test, the $t$-test, and confidence limits on the "estimated model" ( $a$ term to be defined below.) Some of the techniques developed here have analogous developments in system identification literature. ${ }^{6,7}$ We include them here to provide an expanded, unified development and to emphasize that regression diagnostics are among the many statistical techniques that can be applied to the feedforward active control problem.

The link between regression and feedforward control was first noted by Snyder and Hansen, ${ }^{8,9}$ who simulated a feedforward control problem using commercial regression software. Since commercial regression packages operate only on real-valued data, Snyder and Hansen partitioned their problem into separate real and imaginary parts. The present work uses a more general formulation that expresses the regression in the complex domain. The complex formulation allows the use of regression diagnostics and collinearity diagnostics, which were not discussed by Snyder and Hansen. Much of the theoretical development for least- 


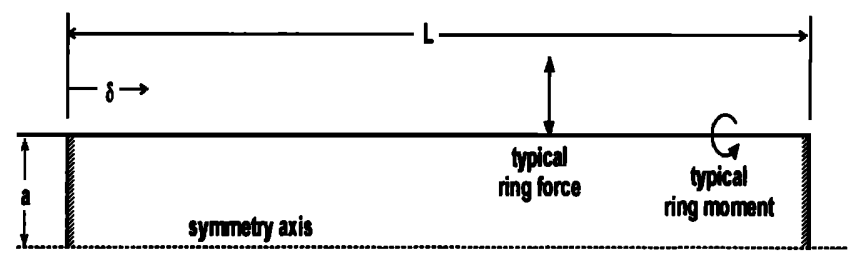

FIG. 1. Geometry of clamped cylindrical shell and typical control inputs.

squares regression of complex variables is taken from Miller. ${ }^{10,11}$

The other major topic of this paper is collinearity, a type of numerical ill conditioning that can corrupt the results of the control simulation. Given that feedforward control in the frequency domain is similar to regression, one might logically assume that numerical difficulties commonly experienced by regressions also appear in feedforward control simulations. This is true of collinearity, the ill conditioning that occurs when one or more transfer functions are not orthogonal. Collinearity is always present to some degree; in fact, control simulations are perhaps more prone to collinearity than other regressions. To simulate a feedforward controller without checking for collinearity is to risk producing meaningless results. The objective is to detect whether the collinearity has serious consequences, and to learn which actuators are causing collinearity problems.

Rosenthal ${ }^{12}$ notes that redundancies between the sensors in an active noise filtering system can produce numerical problems limiting the number of sensors that can be used. The issue is also discussed by Nelson and Elliott ${ }^{1}$ in the context of iterative gradient descent solution methods. Two references that provide a comprehensive discussion of collinearity diagnostics for real-valued regressions are Belsley et al. ${ }^{13}$ and Belsley. ${ }^{14}$ All of the concepts discussed in this paper are based on the techniques in Ref. 14. The extensions to complex-valued regressions are straightforward, and are based on the work of Miller. ${ }^{11}$ The present paper describes how collinearity enters an active control system and how it can affect a feedforward control simulation. Also given is a basic diagnostic procedure for detecting and analyzing collinearity. It is shown that when the sole purpose of the diagnostic is to detect rather than analyze collinearity, the collinearity diagnostic consists of simply examining the condition number of a special scaled design matrix.

While most of the concepts discussed here apply to any feedforward control system, for purposes of illustration we focus on active structural-acoustic control (ASAC). ASAC involves controlling the acoustic response of a fluidstructure system by applying vibrational inputs directly to the structure. Recent theoretical and experimental studies use ASAC to control radiation from one-dimensional and twodimensional structures including beams, plates, and submerged plates; similar techniques are used to control noise transmitted through flat plates or into infinite cylindrical shells. ${ }^{15-24}$

The example structure used for numerical results (Fig. 1) is a finite-length, fluid-loaded cylindrical shell with clamped, rigid, flat end caps. The entire investigation is conducted in the frequency domain, i.e., using a time-harmonic distur- bance whose time dependence $e^{i \omega t}$ is omitted for simplicity of notation. Note that although an axisymmetric example is used here, regression analysis can be applied to threedimensional problems as well. A three-dimensional case is treated in Ref. 25 by superposing the axisymmetric solutions for a spherical shell to obtain a radiated field with a plane of symmetry. Similar techniques may be used with the procedures outlined here.

\section{REGRESSION AND FEEDFORWARD CONTROL}

First we review the fundamentals of multiple linear regression as used in general statistics applications. We then discuss how a feedforward active control problem can be expressed as a complex-valued multiple linear regression, paying special attention to the effects of measurement noise. Finally, we discuss the relation between the weighting function and the physical significance of the cost function, and examine some numerical results for the cylindrical shell example problem. Much of the following discussion may be found in textbooks and articles on linear algebra or leastsquares approximation; see, for example, Refs. 26-29. The discussion is included here to explicitly define the somewhat confusing nomenclature, and to help illustrate the links between feedforward control and regression.

\section{A. Fundamentals of multiple linear regression}

In regression one seeks a model $\bar{Y}\left(\beta_{i}, X_{i}\right)$, the true model, to approximate a set of measured data $Y$, also called the dependent variable. The true model is a function of $\beta_{i}$ (the true regression coefficients) and a collection of predictor variables $X_{i}$, also called independent variables. The data and predictor variables contain $M$ observations, $j=1,2, \ldots, M$, and are written in vector form as $Y=\left\{y_{1} \cdots y_{M}\right\}^{T}$ and $X_{i}=\left\{X_{1 i} \cdots X_{M i}\right\}^{T}$.

Suppose that each observed value $y_{i}$ contains a small amount of Gaussian measurement noise $\epsilon_{i}$. (Note the use of the term "noise" rather than "error." In statistical texts, measurement noise is referred to as "measurement error" or "observation error," whereas in active control literature the term "error" refers to the residual signal that remains after cancellation. To avoid confusion, the term "measurement noise" will refer only to the random component of the measured data $y_{i}$.) Assume also that model we seek (the true model) gives a good approximation of the data:

$$
y_{i}=\tilde{y}_{i}+\epsilon_{i},
$$

where the expected value of the noise is $E\left[\epsilon_{i}\right]=0$. Because we only assume the validity of Eq. (1), we must later test whether the data cause us to reject the assumption.

To use regression diagnostics, we must make some assumptions about the measurement noise. These assumptions are not needed to perform the regression; we can always use regression to find a set of regression coefficients. However, sometimes we wish to go beyond merely computing the regression coefficients, estimated model, and residuals. The assumptions concern $V\left(y_{i}\right)$, the variance of the $i$ th data measurement. A general form for the variance is

$$
V\left(y_{i}\right)=\sigma_{Y}^{2} g^{2}\left(\tilde{y}_{i}, w_{i}\right)
$$


where $\sigma_{Y}^{2}$ is a variance coefficient and $g^{2}\left(\tilde{y}_{i}, w_{i}\right)$ is a variance function. By including $\tilde{y}_{i}$ in the variance function $g^{2}\left(\tilde{y}_{i}, w_{i}\right)$, we allow the possibility that measurement noise may depend on the magnitude of the quantity being measured. The weighting coefficients $w_{i}$ allow us to weight some observations more heavily than others, for example, placing more emphasis on those observations known to be most reliable. Assume that the $\epsilon_{i}$ are normally distributed and pairwise uncorrelated; that is, $\epsilon_{i}$ and $\epsilon_{j}$ are uncorrelated when $i \neq j$. Next we make a simplifying assumption to be scrutinized later: we assume that $V\left(y_{i}\right)$ is independent of $\tilde{y}_{i}$. This can be written

$$
V\left(y_{i}\right)=\frac{\sigma_{Y}^{2}}{w_{i}}, \quad \text { or, equivalently, } \quad V(Y)=\sigma_{Y}^{2} W^{-1},
$$

where we have defined a weighting matrix $W=\operatorname{diag}\left(w_{1}, w_{2}, \ldots, w_{N}\right)$.

Specifying $\sigma_{Y}^{2}$ is critical. Ideally, $\sigma_{Y}^{2}$ should come from some a priori knowledge of the physical measurement system to be used. For example, we could estimate $\sigma_{Y}^{2}$ by repeatedly measuring a calibration signal and computing the sample variance of the measurements. In a preliminary investigation such as the present work, however, we might not know $\sigma_{Y}^{2}$. Lacking a measured value of $\sigma_{Y}^{2}$, the data itself can be used to estimate $\sigma_{Y}^{2}$; this approach is used in the present work. The estimated residual variance $s_{Y-\hat{Y}}^{2}$ [Eq. (23)] is taken as an approximation of $\sigma_{Y}^{2}$.

Let us now assume a linear regression model of the form

$$
\tilde{Y}=\sum_{i=1}^{N} \beta_{i} X_{i}
$$

Because we do not know the true model $\tilde{Y}$, we cannot directly find the true regression coefficients $\beta_{i}$. But we can use $X_{i}$ to compute an estimated model $\hat{Y}$ with estimated regression coefficients $b_{i}$ :

$$
\hat{Y}=\sum_{i=1}^{N} b_{i} X_{i}
$$

The objectives are twofold. First, find predictor variables $X_{i}$ such that the true model $\tilde{Y}$ closely approximates the measured data $Y$. Second, find estimated regression coefficients $b_{i}$ such that the estimated model $\hat{Y}$ closely approximates the true model $\tilde{Y}$. The $F$ test and $t$ test, reviewed in the next section, measure how well we have achieved these objectives.

To simplify notation, we write the estimated regression coefficients in vector form $B=\left\{\begin{array}{llll}b_{1} & b_{2} & \cdots & b_{M}\end{array}\right\}^{T}$. Defining a design matrix $X=\left\{\begin{array}{llll}X_{1} & X_{2} & \cdots & X_{M}\end{array}\right\}$, we can express the estimated model as

$$
\hat{Y}=X B \text {. }
$$

We seek a weighted least-squares solution that minimizes the weighted cost function

$$
\chi^{2}=\sum_{i=1}^{M} w_{i}\left|y_{i}-\hat{y}_{i}\right|^{2}
$$

TABLE I. Comparison of statistics terminology to active control terminology.

\begin{tabular}{ll}
\hline \hline Statistical regression terminology & \multicolumn{1}{c}{ Active control terminology } \\
\hline Dependent variable; data & $\begin{array}{l}\text { inverse of primary response; } \\
\text { inverse of open-loop response } \\
\text { secondary response; control response } \\
\text { Model; estimated model } \\
\begin{array}{l}\text { Independent variable; } \\
\text { predictor variable }\end{array}\end{array}$ \\
$\begin{array}{l}\text { transfer function } \\
\text { Residual }\end{array}$ & $\begin{array}{l}\text { control force; control input } \\
\text { error; closed-loop response; } \\
\text { residual response }\end{array}$ \\
Observation or measurement & error sensor \\
Observation or measurement error & error sensor measurement noise \\
\hline \hline
\end{tabular}

where $y_{i}-\hat{y}_{i}$ is the residual. By requiring the derivatives $\partial\left(\chi^{2}\right) / \partial b_{i}$ to vanish, we obtain the weighted normal equation

$$
X^{T} W X B=X^{T} W Y,
$$

whose solution is the required vector of estimated regression coefficients $B$.

\section{B. Formulating feedforward active control as a complex-valued regression}

First, define the primary response $P_{p}$ as the vector of error sensor outputs due to the disturbance input. Similarly, define a transfer function $X$ as the vector of error sensor outputs that would result from a single actuator of unit strength acting alone. Finally, define the secondary response $\hat{P}$ as the vector of error sensor outputs that would result from all the actuators acting together but without the disturbance.

To formulate the problem as a multiple linear regression, we must have a linear system as defined by three conditions. The response due to a single actuator alone must equal its transfer function multiplied by its complex strength. The secondary response must be a linear sum of all the actuator transfer functions multiplied by their respective complex strengths. Finally, the total or residual response must equal the sum of the primary response plus the secondary response.

Now we can list one by one the elements of the feedforward control problem and their counterparts in the regression problem, which are listed in Table I. The transfer functions are analogous to the predictor variables. The control forces are analogous to the estimated regression coefficients $b_{i}$. The secondary response $\hat{P}$ is analogous to the estimated model $\hat{Y}$. Last, $P=-P_{p}$ (the additive inverse of the primary response) is analogous to the measured data $Y$. By choosing control forces such that $\hat{P}$ approximates $P$, we cause the secondary response to "cancel" the primary response, so that the residual response $P+\hat{P}=-P_{p}+\hat{P}$ is approximately zero.

Recalling that our variables are complex valued, we rewrite the cost function of Eq. (7) as

$$
\chi^{2}=\sum_{i=1}^{M} w_{i}\left(p_{i}+\hat{p}_{i}\right)^{*}\left(p_{i}+\hat{p}_{i}\right)
$$

After algebra, ${ }^{28}$ we can show that the weighted normal equation takes the form

$$
X^{H} W X B=X^{H} W P,
$$


where the superscript $H$ indicates the Hermitian conjugate. Similarly, we must modify how the error sensor measurement noise is characterized. If $\tilde{P}$ is the true model approximated by $\hat{P}$, we now assume that

$$
p_{i}=\tilde{p}_{i}+\epsilon, \quad E\left(p_{i}\right)=\tilde{p}_{i}, \quad \text { and } V\left(\left|\epsilon_{i}\right|\right)=\sigma_{P}^{2} .
$$

In other words, we characterize only the variance of the noise magnitude $|\epsilon|$; we do not specify separate variances for the magnitude and phase. Implications of this relationship are discussed later in the paper.

\section{Physical significance of the cost function}

Often, the cost function approximates a physical quantity. The cost function's physical significance depends on the sensors (predictor variables) and the weighting coefficients. For the example problem in this article, the $M$ sensors are far-field pressure sensors and the predictor variables are vectors of pressure per unit force. The $N$ predictor variables are nothing more than transfer functions between unit actuator forces on the structure and the resulting far-field pressures $P_{j}\left(\theta_{i}\right), i=1,2, \ldots, M, j=1,2, \ldots, N$. Consider the following expressions for the radiated power: ${ }^{30}$

$$
\Pi=\oint_{S^{\prime}} \frac{\pi r^{2}}{\rho c}|P+\hat{P}|^{2} d S^{\prime} \cong \sum_{i=1}^{M} \frac{\pi r^{2} a_{i}}{\rho c}\left|p_{i}+\hat{p}_{i}\right|^{2},
$$

where $S^{\prime}$ is a spherical surface of far-field radius $r$ with the origin at its center, and $a_{i}$ is the area on the surface $S^{\prime}$ associated with the $i$ th far-field location. If $a_{\max }$ is the largest value of $a_{i}$, we can rewrite the estimated radiated power as

$$
\hat{\Pi}=\frac{\pi r^{2} a_{\max }}{\rho c} \sum_{i=1}^{M} \frac{a_{i}}{a_{\max }}\left|p_{i}+\hat{p}_{i}\right|^{2} .
$$

By comparison with Eq. (7), the cost function will be proportional to the radiated power if we specify weighting coefficients of the following form:

$$
w_{i}=a_{i} / a_{\max } .
$$

If there are enough error sensors to completely characterize the radiated field, then the cost function $\chi^{2}$ is proportional to the radiated power $\Pi$; minimizing one minimizes the other. To obtain the radiated power from the cost function, we must use

$$
\hat{\Pi}=\left(\pi r^{2} a_{\max } / \rho c\right) \chi^{2} \text {. }
$$

For the remainder of this text, all references to the total radiated power refer to the estimated radiated power in Eq. (15).

The formulation above places no restrictions on the physical configuration of the control system. The present text discusses an axisymmetric example problem for simplicity of notation, since far-field locations may be specified by only one angle rather than two, but the method is not limited to axisymmetric cases. The transfer functions can contain pressures from any number of sensors in any physical locations desired. Assuming the number of sensors is sufficient, and the sensor locations and weighting coefficients are appropriate, the cost function can approximate the radiated power.

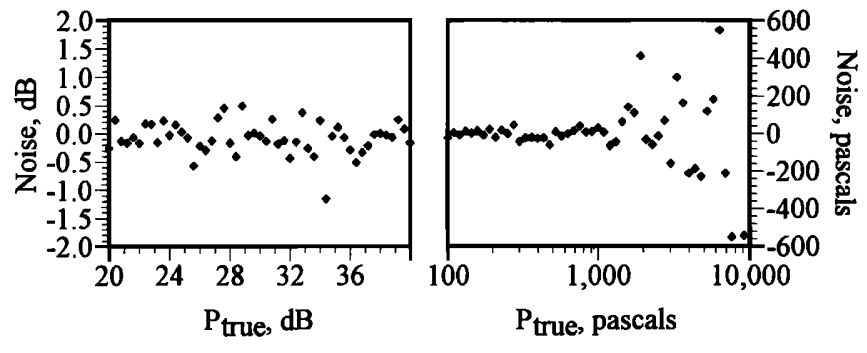

FIG. 2. Example of measurement noise with nonconstant variance. Expressed in decibels, left, the noise variance is independent of signal amplitude. Expressed in linear units, right, the variance is not independent of signal magnitude.

Another important point is that any combination of actuators and sensors can be used. Physical interpretation of the cost function, however, will only be possible with certain combinations of actuators, sensors, and weighting coefficients. Also, sensors need not be distributed throughout the entire radiated field; they could be concentrated in one area to reduce radiation in a certain direction. The reduction of radiated power is a specific case of a more general cost function.

\section{Assumptions regarding variance of measurement noise}

The previous section made some simplifying assumptions about the variance of the measurement noise in a general regression problem; see Eq. (3). Here we examine those assumptions in the context of the ASAC problem to determine whether they represent the type of sensor measurement noise expected in a real control system. Recall that the regression itself does not depend on the distribution of measurement noise, which plays a part only in the regression diagnostics. As discussed below, Eq. (3) may not be entirely appropriate for some types of sensors likely to be used in active noise control systems.

Suppose the error sensors will be hydrophones. Suppose we repeatedly measure a calibration signal $P_{\text {true }}$ and find that the measurements, when expressed in decibels, exhibit Gaussian measurement noise with a sample standard deviation of, say, $s_{p}=0.1 \mathrm{~dB}$. For real hydrophones, this form of approximation may be reasonable. But what if the measurement noise is Gaussian only when expressed in decibels, while we have defined the regression in linear units of pressure? Consider Fig. 2, which shows a hypothetical set of hydrophone measurements of a "known" pressure $P_{\text {true }}$ over a range of pressure levels. The measurement noise is the difference in magnitudes between $\boldsymbol{P}_{\text {true }}$ and the measured value. When the measurement noise is expressed decibels, as in the left plot, the noise variance appears to be constant with respect to the magnitude of $P_{\text {true }}$. But when the same data are plotted in units of pressure instead of decibels, the noise is larger at high pressures than at low pressures. In other words, the measurement noise has a nonconstant variance. To describe the variance properly we must use Eq. (2) rather than using the simplified Eq. (3). 
Because the primary response varies in space, our supposed hydrophone error sensors will measure different pressure magnitudes depending on their locations. A sensor measuring a large pressure will thus have a larger noise component (in linear units of pressure) than a sensor measuring a small pressure. This highlights an important point: the assumptions used to derive Eq. (3) might not provide an accurate representation of the noise present with real sensors. Perhaps the variance should instead be proportional to the signal magnitude as in Eq. (2). For simplicity in the remainder of this paper, we shall assume Gaussian measurement noise with a constant variance as in Eq. (3). The point of the above discussion is that one must carefully evaluate the mathematical form of measurement noise based on the specific sensors being used, and then formulate the regression diagnostics appropriately.

More sophisticated treatments of variance are available, but they are beyond the scope of the present analysis. Most involve performing a transformation and then using of some form of generalized least squares; see Ref. 29. For example, it may be necessary to convert the transfer functions and error sensor measurements to decibels before using them in a regression. In many cases it will likely be seen that the use of standard constant-variance techniques is well justified, and that more complicated techniques may be of limited benefit.

\section{E. The squared multiple correlation coefficient $\mathbf{R}^{2}$}

The squared multiple correlation coefficient $\mathbf{R}^{2}$, commonly used to measure the quality of a regression, has a corresponding role in an active control simulation. For a regression that does not include a constant term as one of the predictor variables, $\mathbf{R}^{2}$ may be written

$$
\mathbf{R}^{2}=\frac{\sum_{j=1}^{M}\left|\hat{p}_{j}\right|^{2}}{\sum_{j=1}^{M}\left|p_{j}\right|^{2}}=1-\frac{\sum_{j=1}^{M}\left|p_{j}-\hat{p}_{j}\right|^{2}}{\sum_{j=1}^{M}\left|p_{j}\right|^{2}},
$$

as described in, for example, Appendix II of Ref. 6. A value of $\mathbf{R}^{2}$ near unity indicates that the estimated model closely fits the measured data. Since $p$ is the open-loop response and $\hat{p}-p$ is the closed-loop response, the controller reduces the open-loop response by an amount expressed in decibels as

$$
\Delta_{\mathrm{dB}}=-10 \log \left(1-\mathbf{R}^{2}\right) .
$$

\section{F. The role of regression diagnostics}

The previous sections describe how a regression predicts control system performance, but do not explicitly consider measurement noise. The regression diagnostics described in this section, which require us to assume a value for the measurement noise variance, provide a more complete picture.

\section{Testing the regression for lack of fit: The F-test}

The " $F$-test" measures confidence in the regression as a whole. The other regression diagnostics which follow all assume that the estimated model closely approximates the true model, a condition that cannot be directly verified because we cannot know the true model. However, if we can show via statistical inference that the variance of the estimated model is not drastically different from the variance of the true model, e.g., that there is no statistically significant lack of fit, then we can proceed with reasonable confidence. The $F$-test verifies whether this condition is true.

The residual signal sensed by the error sensor contains one or more of the following three components: measurement noise, model error, and uncertainty. Measurement noise is always present because the error sensors are imperfect. Model error is present if the disturbance response contains dynamics that are not contained in the control response, i.e., unmodeled dynamics. Uncertainty is present even when there is no model error, because the control forces (regression parameters) cannot be estimated perfectly in the presence of measurement noise. If the model is nearly perfect, that is, if all modes in the disturbance response are adequately represented in the control response, then the residual contains only measurement noise and uncertainty. There is no lack of fit, and the residual is the smallest residual that can be expected based on the amount of measurement noise introduced by the sensors. On the other hand, if there are significant unmodeled dynamics, there is a lack of fit and the residual will be larger than the minimum possible residual. The $F$-test gives a statistical measure, based on the data, of whether there is a statistically significant lack of fit.

Begin by assuming that there is no lack of fit, and thus the control response $\hat{P}$ is close to the true model $\tilde{P}$; call this assumption $H_{0}: \hat{P} \approx \tilde{P}$. One way of verifying $H_{0}: \hat{P} \approx \tilde{P}$ is to show that $\hat{P}$ and $\tilde{P}$ have the same variance:

$$
V(\bar{P}-\bar{P}) \approx V(\hat{P}-\bar{P}),
$$

where $\bar{P}$ is the estimated mean of the disturbance response. Since we cannot know $\tilde{P}$ directly, we use a relation from regression theory, ${ }^{27}$

$$
V(\bar{P}-\bar{P})=V(P-\hat{P})+V(\hat{P}-\bar{P}) .
$$

Loosely speaking, Eq. (19) states that the variance of the true model about $\bar{P}$ equals the residual variance plus the variance due to the regression. We can rearrange Eq. (19) as

$$
V(\bar{P}-\bar{P})=V(\hat{P}-\bar{P})\left\{1+\frac{1}{F}\right\}, \quad \text { where } F=\frac{V(\hat{P}-\bar{P})}{V(P-\hat{P})}
$$

and $F$ is the so-called variance ratio. To satisfy Eq. (18) as closely as possible we must have $F \gg 1$, in which case we do not reject $H_{0}: \hat{P} \approx \tilde{P}$. The $F$-test is not violated, and there is no significant lack of fit.

To determine whether $F$ is large enough, we compare $F$ to a predetermined value. Let $Q\left(F, \nu_{1}, \nu_{2}\right)$ be the $F$-distribution probability function with $\nu_{1}$ degrees of freedom in the numerator and $\nu_{2}$ degrees of freedom in the numerator. Then, given $X, P, M$, and $N$ for the specific problem at hand, $Q\left(F, \nu_{1}, \nu_{2}\right)$ is the probability of a statistically significant lack of fit, that is, the probability that $F$ could be as large as it is by chance alone rather than because of the regression. When $Q\left(F, \nu_{1}, \nu_{2}\right)$ exceeds some specified risk level, we must reject $H_{0}: \hat{P} \approx \tilde{P}$. In the present context with $N$ actuators and $M$ sensors, we have

$$
\nu_{1}=N \text { and } \nu_{2}=M-N-1 .
$$


Values for $Q\left(F, \nu_{1}, \nu_{2}\right)$ are easily generated by computer or read from tables. The variance ratio can be approximated by the ratio of standard deviations

$$
F=s_{\hat{P}-\bar{P}}^{2} / s_{P-\hat{P}}^{2}
$$

where

$$
s_{\hat{P}-\bar{P}}^{2}=\frac{1}{\nu_{1}} \sum_{i=1}^{M} w_{i}\left|\hat{p}_{i}-\bar{p}\right|^{2}
$$

$$
s_{P-\hat{P}}^{2}=\frac{1}{\nu_{2}} \sum_{i=1}^{M} w_{i}\left|p-\hat{p}_{i}\right|^{2} .
$$

If $F$ is large enough, that is, if $Q\left(F, \nu_{1}, \nu_{2}\right)$ does not exceed the specified acceptable risk level, then there is no lack of fit and we can proceed with testing the individual regression coefficients as described in the next section. If $F$ is too small, then it is too likely that we could have chosen regression coefficients at random and still achieved the same fit to the data.

When a regression fails the $F$-test, it generally means this: Given $X, P, M$, and $N$, there is too much variance in the measured data $P$ for us to confidently say that we have modeled the data. In terms of an ASAC problem, one or more of the following conditions exists:

(1) There are too few sensors to confidently say that the response has been adequately modeled, i.e., the data and predictor variables are undersampled. Adding more sensors will provide better estimates of the variances, and may increase $F$ unless other problems are present.

(2) The actuators are decoupled from one or more modes that are present in the disturbance response, i.e., the disturbance response contains unmodeled dynamics that cannot be reproduced by the actuators. Adding more actuators or using different actuators that couple into different modes will improve controllability.

(3) The amount of measurement noise present is much greater than originally thought. Even if the actuators couple into the same modes that appear in the disturbance response, severe measurement noise can have the same effect as unmodeled dynamics.

Generally speaking, a regression will only fail the $F$ test when the control differs substantially from the disturbance response. Any sound cancellation is coincidental and occurs only in localized areas rather than globally.

\section{The t-test and confidence limits for individual regression coefficients}

The $t$-test, which examines the individual regression coefficients corresponding to the control inputs in the active control problem, allows us to detect whether any actuators are completely uncoupled from the cost function. If a given actuator has no beneficial effect on the regression, then its regression coefficient vanishes and we could obtain a more reliable regression by reformulating the problem without that actuator. However, determining whether a given coefficient

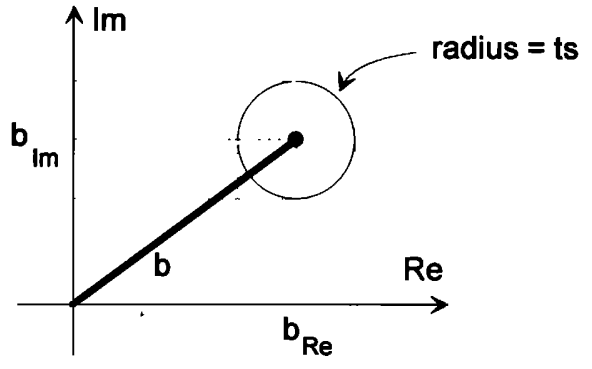

FIG. 3. Circular confidence limit on a regression parameter, where $\operatorname{Re}\{b\}=b_{\mathrm{Re}}$ and $\operatorname{Im}\{b\}=b_{\mathrm{Im}}$. The confidence limit specifies a circular region of radius $t s_{b}$ centered at $\left(b_{\mathrm{Re}}, b_{\mathrm{Im}}\right)$ such that the true parameter lies within the circle.

is zero can be difficult because problem scaling may yield arbitrarily small coefficients. To decide whether a coefficient should be removed, we can define a statistical "confidence interval" for the magnitude of that coefficient, and then check whether the confidence interval contains zero.

Assuming Gaussian measurement noise, the magnitude variance of the $i$ th control force is

$$
\sigma_{b_{i}}=\sqrt{e_{i}},
$$

where $e_{i}$ is the $i$ th diagonal of the variance-covariance matrix

$$
V(b)=\sigma_{p}^{2}\left(X^{H} W X\right)^{-1}
$$

and $\sigma_{p}$ is the variance of the measurement noise. Since the true error variance $\sigma_{p}$ is usually not known, we can use the estimated variance $s_{p}$ in its place to obtain an estimated variance $s_{b_{i}}$.

If an estimated noise variance is available for a specific type of error sensor, that variance may be used in performing a simulation. If no such estimate is available, the residual variance $s_{\hat{P}}-\bar{P}$ may be used instead. Because the present work emphasizes numerical methods rather than results for specific types of hardware, all numerical results in this paper use error variances that are estimated from the data. The reader is reminded that a specific value of the error variance is required only for regression diagnostics, not for the regression itself.

Next we decide on a level of statistical significance $\alpha_{t}$, say $90 \%$, and compute a quantity known as Student's $t$, the value of the Student's probability distribution for the chosen level of significance $\alpha_{t}$ and $\nu$ degrees of freedom, where $\nu=M-N-1$. We can then state that given the data, the probability of $\left|b_{i}-\beta_{i}\right|<t s_{b_{i}}$ is $\alpha_{t}$. In other words, there is a $90 \%$ probability that the magnitude of the error between the true control input and that computed by the regression is smaller than $t s_{b_{i}}$. The confidence interval provides a circular confidence region on $b_{i}$ in the real-imaginary plane (Fig. 3) such that

$$
\left|b_{i}\right|-t s_{b_{i}} \leqslant\left|\beta_{i}\right| \leqslant\left|b_{i}\right|+t s_{b_{i}} .
$$

The largest magnitude inside the confidence region, which represents the largest likely control input magnitude, is $\left|b_{i}\right|+t s_{b_{i}}$. As noted in Ref. 7, we could also consider the 
real and imaginary parts of the problem separately to produce an elliptical confidence region.

A related statistical test is the so-called " $t$-test," which measures whether a given control input is significantly different from zero. If the range $\left\{\left|b_{i}\right|-t s_{b_{i}},\left|b_{i}\right|+t s_{b_{i}}\right\}$ contains zero at the specified level of significance, then we cannot conclude that $b_{i}$ is significantly different from zero. Said another way, if an actuator "fails the $t$-test," we have no statistically significant indication that control input has nonzero magnitude. Adding sensors for a given number of actuators has the effect of decreasing $t$, and thus producing tighter confidence intervals and a more reliable model. In terms of the ASAC problem, a control input will only be set equal to zero if the actuator is completely uncoupled from the disturbance response. Therefore any actuator that fails the $t$ test should be removed from the problem.

\section{Prediction intervals}

The confidence intervals for all the actuators may be condensed into a single quantity, the prediction interval, which provides a confidence interval on the magnitude of the control response $\hat{P}$. The regression provides an estimated residual $\boldsymbol{P}+\hat{P}$ corresponding to the pressure when the control actuators are operating. The control response $\hat{P}$ is based on error sensor outputs that contain measurement noise, and its reliability is therefore related to the accuracy of the error sensors. By applying the appropriate regression diagnostics, we can estimate confidence intervals on $\hat{P}$ and provide a single quantity that reflects the sensitivity to measurement noise for the regression as a whole.

At a given sensor location, the control response is a linear sum:

$$
\hat{P}_{j}=\sum_{i=1}^{n} b_{i} X_{i j},
$$

where the control inputs $b_{i}$ have variances as expressed as in the previous section, and the transfer functions $X_{i}$ are considered constant and completely accurate for the present purposes. If we define the vector $X_{i, 1: N}$ $=\left\{\begin{array}{llll}X_{i, 1} & X_{i, 2} & \cdots & X_{i, N}\end{array}\right\}$ as the $i$ th row of the design matrix $X$, it can be shown that the variance of the estimated model at the location of the $j$ th sensor is

$$
V\left(\hat{P}_{j}\right)=\sigma_{P}^{2} X_{i, 1: N}\left(X^{H} W X\right)^{-1} X_{i, 1: N}^{H} .
$$

This is also equal to the $j$ th diagonal of the variancecovariance matrix

$$
V(\hat{P})=\sigma_{P}^{2} X\left(X^{H} W X\right)^{-1} X^{H} .
$$

Using Student's $t$ for a chosen level of significance, the prediction interval for the estimated model at the $j$ th sensor location is defined by the standard error

$$
\pm t \sigma_{\bar{P}+P} \sqrt{V\left(\hat{P}_{j}\right)} \text {. }
$$

The prediction interval summarizes the sensitivity to measurement noise for the entire regression, whereas the confidence intervals address individual actuators. Prediction intervals can be used to determine whether a given combina-

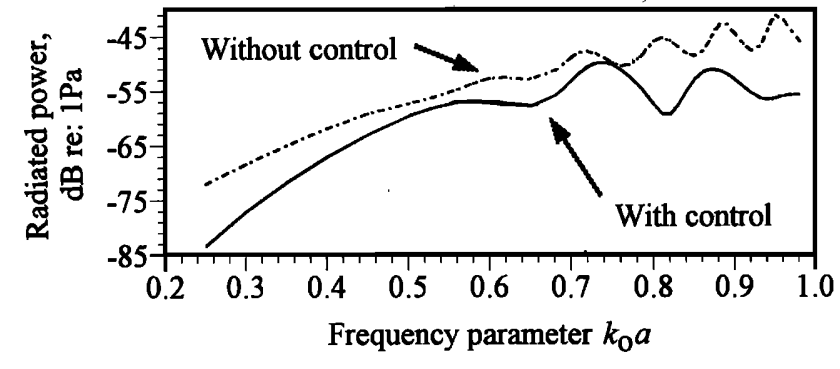

FIG. 4. Radiated power versus frequency. Radiated power without control actuators shown as solid line; with control actuators shown as broken line.

tion of structure, actuators, and sensors is particularly sensitive to noise and, if so, what portions of the radiated field are most likely to be degraded by noise. As with confidence intervals, increasing the number of sensors for a given number of actuators has the effect of decreasing the width of the prediction interval.

\section{G. Numerical results and discussion}

The example structure used for numerical results is a finite-length, fluid-loaded cylindrical shell with rigid, flat end caps (Fig. 1). The points at which the cylinder intersects the end closures are constrained both in translation and in rotation, i.e., the cylindrical section is clamped. The shell length $L$ is $10 \times$ the shell radius $a$, and the ratio of wall thickness to shell radius is 0.005 . The global $z$ axis is the shell symmetry axis, with the right-hand end cap intersecting the positive $z$ axis. Far-field locations are described by the global spherical coordinates $(R, \theta, \phi)$ with origin at the midlength of the shell, where the far-field location $\theta=0$ corresponds to the positive $z$ axis. Surface locations are described by a normalized distance coordinate $\delta=z / L$. We examine frequencies as high as $k_{0} a=0.95$. Material properties are chosen to resemble an undamped steel shell submerged in water: the shell material has Young's modulus of $1.85 \times 10^{11} \mathrm{~Pa}$, Poisson's ratio of 0.3 , and density of $7670 \mathrm{~kg} / \mathrm{m}^{3}$. The density and acoustic velocity of the surrounding fluid are $1000 \mathrm{~kg} / \mathrm{m}^{3}$ and $1460 \mathrm{~m} / \mathrm{s}$, respectively. All far-field quantities are normalized to a distance of $1.0 \mathrm{~m}$. Numerical solutions for structural-acoustic dynamic response are found using the combined finite-element/boundary-element computer program NASHUA. ${ }^{31}$

The cylinder is disturbed by a unit-magnitude axisymmetric ring force $b_{d}$ placed near the right-hand end closure at $\delta=0.96$. The two actuators $(M=2)$ are axisymmetric ring force $b_{1}$ and $b_{2}$ placed at $\delta=0.25$ and $\delta=0.50$. The 25 error sensors $(N=25)$ are pressure sensors located at $7.5^{\circ}$ intervals from $0^{\circ}$ to $180^{\circ}$ in the far field. The control objective is to minimize the radiated power cost function $\Pi$, where $\Pi$ is the weighted sum of squared pressure magnitudes at the 25 error sensors.

Figure 4 shows the radiated power cost function $\Pi$ versus nondimensional frequency $k_{0} a$ for the frequency range $0.2<k_{0} a<1.0$. The upper curve represents the open-loop case. The lower curve represents the closed-loop case, and the difference between the two is the reduction in the radiated power cost function. The actuator magnitudes and 


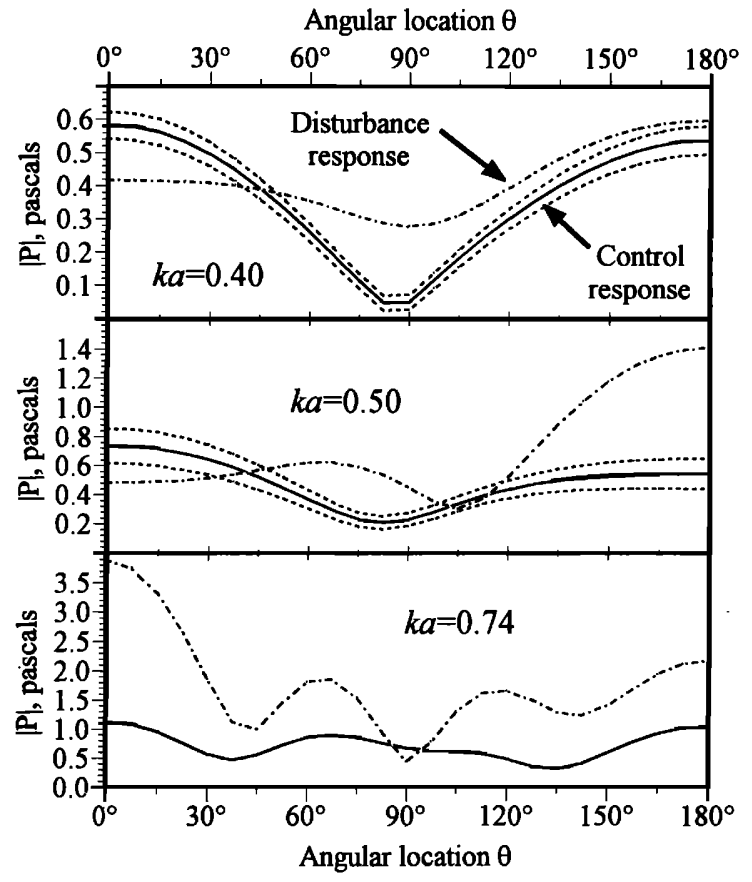

FIG. 5. Confidence limits for estimated control response at three frequencies. Estimated control response pressure magnitude is shown with $90 \%$ confidence limits based on estimated error variance. (Confidence limits omitted for $k_{0} a=0.74$.)

phases have been optimized separately at each frequency in the region, since each regression uses data for a single frequency. The actuators reduce the radiated power cost function by as much as $14 \mathrm{~dB}$ depending on the frequency. Detailed analysis of the structural and acoustic responses before and after applying control is not examined here. Since the intent is to illustrate the use of regression diagnostics, we restrict our attention to three specific frequencies as described in the following paragraphs.

At the first frequency, $k_{0} a=0.40$, the regression predicts that the radiated power cost function will be reduced by $5 \mathrm{~dB}$; the control inputs are $b_{1}=1.06 \mathrm{~N}$ and $b_{2}=-1.08 \mathrm{~N}$. The $F$-test yields $Q\left(F, \nu_{1}, \nu_{2}\right)=1.6 \times 10^{-6}$, which means: given the data, there is only a $0.00016 \%$ probability of a statistically significant lack of fit. We therefore feel confident enough about the regression to continue with further tests.

To use the $t$-test and other diagnostics, we must estimate the measurement noise variance; as in previous sections, we estimate the variance from the disturbance response data. Computing confidence intervals at the $90 \%$ significance level, we find that the control inputs at this frequency are $b_{1}=1.06 \pm 0.27 \mathrm{~N}$ and $b_{2}=-1.08 \pm 0.28 \mathrm{~N}$. Since neither confidence interval contains zero, we can say with $90 \%$ confidence that, given the data and our estimate of the error variance, both control inputs are significantly different from zero. The upper plot in Fig. 5 shows the magnitude of the control response as a function of far-field location, with prediction intervals at the $90 \%$ significance level; also shown is the disturbance response. The average width of the prediction interval is roughly $0.06 \mathrm{~Pa}$, while the signal levels are on the order of $0.6 \mathrm{~Pa}$.

Next, consider $k_{0} a=0.50$. The regression indicates that

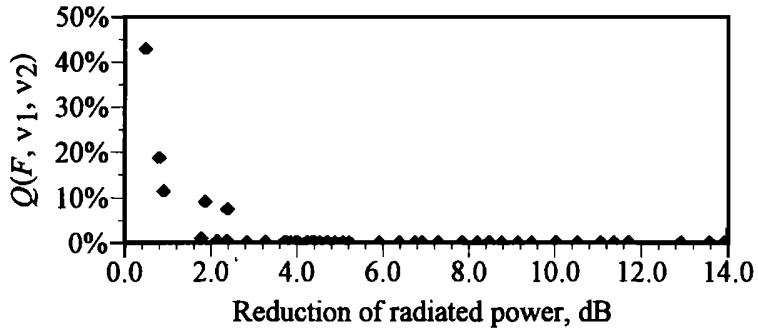

FIG. 6. F-test probability versus reduction of radiated power. Each data point represents a different frequency in the range $0.2<k_{0} a<1.0$. When $Q\left(F, \nu_{1}, \nu_{2}\right)$ is greater than a specified acceptable risk level, say, $\alpha_{t}=1 \%$, there is a statistically, significant lack of fit.

the control inputs $b_{1}=0.61 \mathrm{~N}$ and $b_{2}=0.01 \mathrm{~N}$ reduce the radiated power cost function by about $2 \mathrm{~dB}$. We find that $Q\left(F, \nu_{1}, \nu_{2}\right)=0.0041$, e.g., there is less than one-half percent probability that this solution arose by chance, so we shall consider the regression statistically significant. However, one of the control forces fails the $t$-test: we find that the confidence interval $b_{2}=0.01 \pm 0.31 \mathrm{~N}$ contains zero, and thus we cannot conclude that $\left|b_{2}\right|$ is significantly different from zero. The middle plot in Fig. 5 shows the prediction interval. At this frequency the average width of the prediction interval is $0.08 \mathrm{~Pa}$, larger than for the frequency discussed above. To improve the reliability of the regression, we could reformulate it without $b_{2}$.

Now consider a third single-frequency case with $k_{0} a=0.74$. At this frequency the regression predicts controller performance of less than $1 \mathrm{~dB}$. Performing the $F$-test, we find that $Q\left(F, \nu_{1}, \nu_{2}\right)=0.114$. In other words, there is an $11 \%$ chance that the control response is statistically different from the disturbance :response. The regression at this frequency fails the $F$-test by most reasonable standards, and therefore we cannot make use of regression diagnostics such as confidence intervals and prediction intervals. An examination of the data and the estimated model, shown in the lower plot of Fig. 5, confirms that there is little relation between the control response and the disturbance response. Significant cancellation occurs by chance in a localized area near $\theta=90^{\circ}$, but in general the two curves have very different characteristics.

Figure 6 explores the relationship between the $F$-test results and controller performance. Each data point represents a regression at a different frequency. The quantity $Q\left(F, \nu_{1}, \nu_{2}\right)$ is shown on the vertical axis, where $Q\left(F, \nu_{1}, \nu_{2}\right)$ is the probability that there is a significant lack of fit. Wherever the radiated power cost function is reduced by roughly $2 \mathrm{~dB}$ or more, $Q\left(F, \nu_{1}, \nu_{2}\right)$ is less than $1 \%$ and there is no lack of fit. Only when the radiated power cost function is reduced by less than $2 \mathrm{~dB}$ does $Q\left(F, \nu_{1}, \nu_{2}\right)$ rise to appreciable levels. This result is consistent with other force configurations investigated but not shown here. Failing the $F$-test is always accompanied by poor controller performance, meaning that any sound cancellation is coincidental and probably localized. Likewise, excellent controller performance always signals that the regression has passed the $F$ test. In the present example, a threshold of roughly $2 \mathrm{~dB}$ corresponds to passing or failing the $F$-test. However, it is 


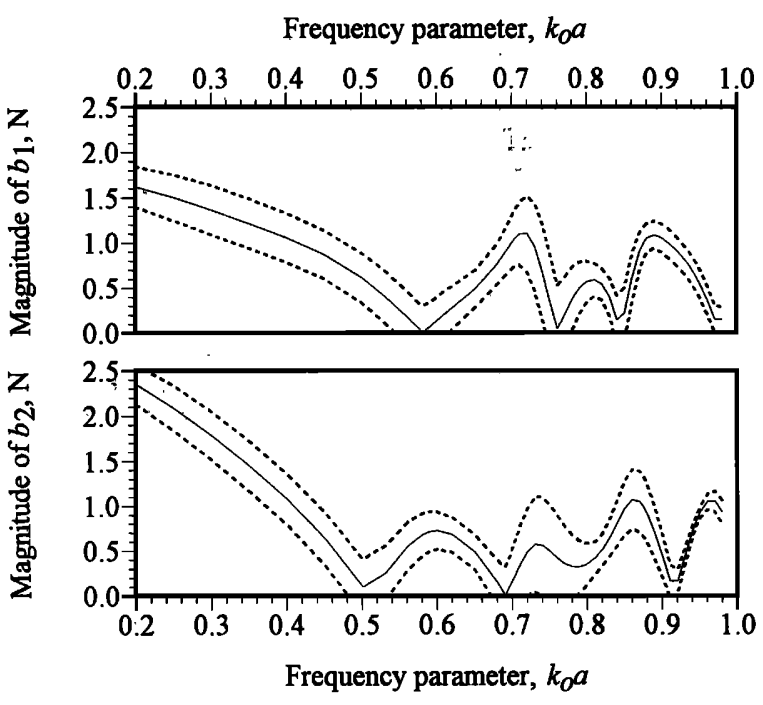

FIG. 7. Magnitude of control forces versus frequency, with $90 \%$ confidence limits on control force magnitude.

difficult to make $a$ priori assumptions about $F$-test results: the threshold is problem specific and can only be found by examining $F$-test results for a number of different frequencies. Other cases not shown here achieved reductions of 4-5 dB but still failed the $F$-test.

Figure 7 shows the magnitudes and magnitude confidence intervals of the two control forces as a function of frequency. The solid curves represent the control force magnitudes, with the upper and lower $90 \%$ confidence limits displayed as broken curves. The upper and lower plots represent $b_{1}$ and $b_{2}$, respectively. There are several frequency ranges in which one of the control forces fails the $t$-test, meaning that one of the confidence intervals contains zero. For example, the confidence interval for $b_{2}$ contains zero near $k_{0} a=0.50, k_{0} a=0.69$, and $k_{0} a=0.91$. When a confidence interval contains zero, the associated control force does not contribute significantly to the regression at that frequency and could probably be removed without significantly affecting the results.

\section{COLLINEARITY AND COLLINEARITY DIAGNOSTICS}

The previous section describes regression and regression diagnostics, and outlines their relationship to simulating a feedforward control system. The topic of this section is collinearity, a type of numerical ill conditioning that can affect the simulation results. Included is a brief example to show how collinearity can affect the results of a numerical controller simulation.

\section{A. How collinearity occurs in active noise control}

Imagine an ASAC problem with two control forces applied at exactly the same location on the structure. In the nomenclature of the previous section, the two transfer functions are identical, the normal equations are singular, and there is no unique solution for the control force magnitudes. Now suppose the two control forces are close together, but not quite collocated. The two transfer functions are not identical, but very similar; the normal equations are not singular,
TABLE II. Effect of simulated measurement noise without collinearity.

\begin{tabular}{ccccccc}
\hline \hline & \multicolumn{2}{c}{ No simulated noise } & \multicolumn{2}{c}{$0.5 \%$ simulated noise } & \multicolumn{2}{c}{$5 \%$ simulated noise } \\
& $b_{i}$ & $\Delta\left|b_{i}\right|$ & $b_{i}$ & $\Delta\left|b_{i}\right|$ & $b_{i}$ & $\Delta\left|b_{i}\right|$ \\
\hline$b_{1}$ & $(-18.1,-0.8)$ & $\ldots$ & $(-18.0,-0.8)$ & $<0.5 \%$ & $(-17.7,-0.8)$ & $2 \%$ \\
$b_{2}$ & $(-21.3,-2.2)$ & $\ldots$ & $(-21.3,-2.2)$ & $<0.5 \%$ & $(-21.0,-2.2)$ & $1 \%$ \\
$b_{3}$ & $(131.9,11.4)$ & $\cdots$ & $(131.9,11.4)$ & $<0.5 \%$ & $(131.8,11.3)$ & $1 \%$ \\
\hline \hline
\end{tabular}

but may be ill conditioned. In fact, the possibility for such near dependencies to cause ill conditioning exists whenever the transfer functions are not all mutually orthogonal.

Suppose we plunge ahead and solve the normal equation; what sort of solution can we expect? Often the adjacent (and partially redundant) control forces have opposite phases and very large magnitudes. In some circumstances such results are purely a numerical artifact, the result of ill conditioning of the normal equation. In other circumstances the results could be interpreted as a force couple approximating a concentrated moment, with no indication of whether the solution was genuine or spurious. This disturbing ambiguity motivates the use of collinearity diagnostics.

Note that control forces need not be located next to each other to produce collinearity. If the modal density is low and the structure is very lightly damped, there may be several locations at which applied forces would produce nearly the same response. Control forces applied at two or more such locations would produce collinearity even though they were distant from each other. Another source of collinearity arises when one control force can be replaced by a linear combination of other control forces in the system.

\section{B. An example showing the effects of collinearity}

An example shows how collinearity can affect even a relatively simple control simulation. Consider the clamped cylinder of Fig. 1 with a disturbance force $b_{d}$ acting at a frequency of $k_{0} a=0.95$. Choose three control actuators $b_{1}$, $b_{2}$, and $b_{3}$, whose locations we shall discuss after performing a brief numerical experiment. The regression predicts controller performance of $23 \mathrm{~dB}$. Since the $F$-test and $t$-test results (not shown) appear reasonable, we accept as significant the following complex control inputs:

$$
\begin{aligned}
& b_{1}=(-18.1,-0.8), \quad b_{2}=(-21.3,-2.2), \\
& b_{3}=(131.9,11.4),
\end{aligned}
$$

where, for example, $\operatorname{Re}\left\{b_{1}\right\}=-18.1$ and $\operatorname{Im}\left\{b_{1}\right\}=-0.8$.

A simple numerical experiment can show how measurement noise in the sensor data $Y=\left\{y_{1}, y_{2}, \ldots, y_{M}\right\}$ affects the regression results. We simulate measurement noise by artificially injecting "random components into $Y$, recomputing the regression, and examining the control input magnitudes. The results are summarized in Table II. The first pair of columns, labeled "No simulated noise," contains the original regression results given in Eq. (31). Next, each error sensor output $y_{i}$ is perturbed with $0.5 \%$ simulated measurement noise, that is, a noise component with random phase and with random magnitude up to $0.5 \%$ of the maximum value in $Y$, and the regression is recomputed using the perturbed $Y$. As shown in the second pair of columns in Table II, simulated measure- 
TABLE III. Effect of simulated measurement noise with collinearity.

\begin{tabular}{ccccccr}
\hline \hline & \multicolumn{2}{c}{ No simulated noise } & \multicolumn{2}{c}{$0.5 \%$ simulated noise } & \multicolumn{2}{c}{$5 \%$ simulated noise } \\
& $b_{i}$ & $\Delta\left|b_{i}\right|$ & $b_{i}$ & $\Delta\left|b_{i}\right|$ & $b_{i}$ & $\Delta\left|b_{i}\right|$ \\
\hline$b_{1}$ & $(-627.7,-37.0)$ & $\ldots$ & $(-604.5,-49.0)$ & $5 \%$ & $(-195.1,-78.9)$ & $67 \%$ \\
$b_{2}$ & $(613.5,36.1)$ & $\ldots$ & $(589.7,48.5)$ & $2 \%$ & $(164.9,78.2)$ & $70 \%$ \\
$b_{3}$ & $(117.3,9.6)$ & $\ldots$ & $(118.1,9.4)$ & $1 \%$ & $(128.2,9.6)$ & $9 \%$ \\
$b_{4}$ & $(24.1,1.4)$ & $\cdots$ & $(23.2,1.9)$ & $2 \%$ & $(7.0,3.1)$ & $68 \%$ \\
\hline \hline
\end{tabular}

ment noise of $0.5 \%$ does not significantly change the predicted control inputs. Next we increase the simulated measurement noise to $5 \%$ and recompute the regression. As shown in the third pair of columns in Table II, the control input magnitudes are changed by less than $2 \%$. In fact, we can increase the simulated measurement noise to $25 \%$ or more before the control inputs change substantially. The regression is relatively insensitive to measurement noise.

Now suppose that we add a fourth control input $b_{4}$ in an attempt to improve system performance. (For the sake of illustration we choose a particularly bad location for $b_{4}$.) For the new configuration with no simulated measurement noise, we predict that the control inputs reduce the radiated power by $29 \mathrm{~dB}$, an improvement of $6 \mathrm{~dB}$ over the previous case. Again the $F$-test and $t$-test results appear reasonable, so we accept as significant the control inputs shown in the first pair of columns in Table III. But now, simulated measurement noise of only $0.5 \%$ produces changes of up to $5 \%$ in the predicted control inputs. If we increase the simulated measurement noise to $5 \%$, the control inputs are completely changed. Still, the $F$-test and $t$-test show no signs of trouble, and the subroutine used to solve the normal equation gives no warning or error messages. Why is the solution so sensitive to measurement noise? The reason, of course, is collinearity between the particular control forces we chose.

Examining the locations of the control inputs, shown in Fig. 8, we can clearly explain the numerical difficulties. The first two inputs $b_{1}$ and $b_{2}$ are axisymmetric ring forces and $b_{3}$ is a distributed axisymmetric ring force. But the control input used in the second example, $b_{4}$, is an axisymmetric ring moment applied exactly between $b_{1}$ and $b_{2}$. The two ring forces, when combined with opposite phases, produce a force couple that has nearly the same effect as the ring moment. The ring moment is redundant, and in this simple example is easily seen as the cause of the numerical problems. The collinearity diagnostics presented in the next section provide a general method that could be used to detect and analyze collinearities in more complicated systems where relations between actuators might not be so clear.

\section{A collinearity diagnostic}

Rather than trying to present a comprehensive review of collinearity diagnostics and their possible applications in studying feedforward control, this section presents one diagnostic method with some basic example results. For more detail the reader is referred to Belsley. ${ }^{14}$

Recall the normal equation for a feedforward control system with $M$ sensors and $N$ actuators,

$$
\left(X^{H} W X\right) B=X^{H} W P \text {. }
$$

As the reader might expect, most collinearity diagnostics involve the condition number of $X^{H} W X$. Clearly a large condition number announces the presence of ill conditioning, but simply examining the condition number leaves three questions unanswered. First, how can we detect whether more than one near dependency is present? Second, how large a condition number can we tolerate and still have confidence in the regression results? And third, how can we determine which actuators are involved in near dependencies? We will briefly discuss these three questions before presenting the diagnostic method.

The first question, regarding the number of near dependencies present, is answered by examining not just the condition number of $X^{H} W X$ but the singular values of $X^{H} W X$. A zero singular value indicates a perfect linear dependency in $W^{1 / 2} X$; a "small" singular value indicates a near dependency that may cause conditioning problems. In fact, the number of small singular values indicates the number of near dependencies present in the problem.

The second question is, how do we make quantitative comparisons to determine whether the condition number is "large," or equivalently, whether a given singular value is small? The answer is that we must scale the columns of $W^{1 / 2} X$ before computing the singular values. Column scaling, described in the next section, permits such quantitative comparisons.

To answer the third question of determining which variables are involved in near dependencies, we turn to a result from the least-squares regression. One of the effects of collinearity is variance inflation: when two or more actuators are involved in a near dependency, their variances become large. We can express the variances in terms of the singular
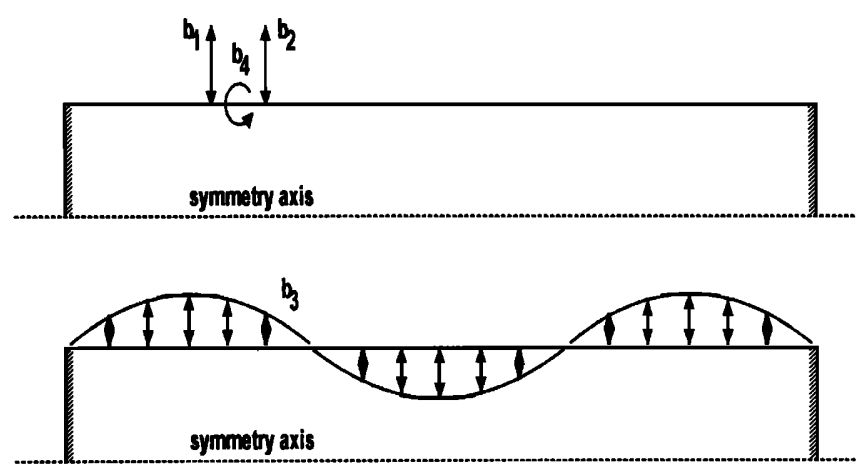

FIG. 8. Example problem for collinearity diagnostics. The first two actuators are axisymmetric ring forces $b_{1}$ and $b_{2}$ applied at $\delta=0.25$ and 0.31 . The third actuator is a axisymmetric distributed force $b_{3}=\cos (3 \pi \delta)$. The fourth actuator is an axisymmetric ring moment $b_{4}$ applied at $\delta=0.31$. 
values of $W^{1 / 2} X$. When we find two or more actuators whose variances are primarily associated with a small singular value, then those actuators are involved in a near dependency.

We now describe the diagnostic method in somewhat of a cookbook fashion. The intent is not to present a detailed derivation, but rather to outline the method and show a simple numerical example related to feedforward control. We modify the technique slightly to account for complex-valued variables and weighted least squares, which are not included in the discussion of Ref. 14.

\section{Determine $W^{1 / 2} X$}

Each column of $W^{1 / 2} X$ represents a transfer function between a given control input and the error sensor array. These may be obtained via analytical approximations, numerical calculations, or any other means available. For $N$ actuators and $M$ sensors, $X$ has the form $X=\left\{\begin{array}{lllll}X_{1} & X_{2} & \ldots & X_{M}\end{array}\right\}$, where each $X_{j}$ is a complex vector of length $M$ containing the transfer function for the $j$ th actuator. In our example the columns of $X$ are transfer functions between four control inputs $(N=4)$ and 25 sensor locations $(M=25)$. We obtain the transfer functions by numerical modeling of the structure using a finite-element/boundaryelement approach.

\section{Apply column equilibration to $W^{1 / 2} X$}

The purpose of column equilibration is to produce a set of singular values that do not depend on the units of the problem. We scale each column in $W^{1 / 2} X$ to have unit vector length after scaling. This produces a new matrix $\mathbf{Z}$, the scaled weighted design matrix:

$$
\mathbf{Z}=\mathbf{\Omega}^{-1 / 2} W^{1 / 2} X,
$$

where $\boldsymbol{\Omega}^{-1 / 2}$ is a diagonal matrix whose diagonals are $\boldsymbol{\Omega}_{j}^{-1 / 2}=\left\{X_{j}^{H} W X_{j}\right\}^{-1 / 2}$. The relation between $\mathbf{Z}$ and the original problem may be seen from

$$
X^{H} W X=\mathbf{Z}^{H} \mathbf{\Omega Z} \text {. }
$$

\section{Obtain scaled condition indexes and variance-decomposition proportions}

First we generate the singular-value decomposition of $\mathbf{Z}$,

$$
\mathbf{Z}=\mathbf{U} D \mathbf{V}^{H},
$$

where $\mathbf{U}$ is column orthogonal, $\mathbf{V}$ is column orthogonal and row orthogonal, and $D$ is nonnegative and diagonal. (Note the distinction between the matrix $\mathbf{V}$ and the variance $V$ ). The elements of $D$ are the singular values $\mu_{k}, k=1,2, \ldots, N$, which are real and non-negative since $\mathbf{Z}$ is Hermitian. The scaled condition indexes are then defined as

$$
\tilde{\eta}_{k}=\mu_{\max } / \mu_{k}, \quad k=1,2, \ldots, N .
$$

If we express the elements of the matrix $\mathbf{V}$ as $\boldsymbol{\nu}_{k j}$, we can write the variances of the regression coefficients in the following useful form:
TABLE IV. Variance decomposition proportions for example problem.

\begin{tabular}{rrrrcc}
\hline \hline$j$ & $\tilde{\eta}_{j}$ & $\pi_{j 1}$ & $\pi_{j 2}$ & $\pi_{j 3}$ & $\pi_{j 4}$ \\
\hline 1 & 1.0 & 0.000 & 0.000 & 0.000 & 0.000 \\
2 & 1.1 & 0.000 & 0.000 & 0.000 & 0.000 \\
3 & 6.3 & 0.000 & 0.000 & 0.871 & 0.000 \\
4 & 11502.1 & 0.999 & 0.999 & 0.129 & 0.999 \\
\hline \hline
\end{tabular}

$$
V\left(b_{k}\right)=\sigma_{P}^{2} \sum_{j=1}^{N} \frac{\nu_{k j}^{2}}{\mu_{j}^{2}} .
$$

The utility of Eq. (37) lies in the fact that each term in the summation is associated with only one scaled condition index $\tilde{\eta}_{k}$. By noting which of the large scaled condition indexes affect which variances, we can pick out near dependencies among the columns of $W^{1 / 2} X$. To this end, let us define variance-decomposition proportions,

$$
\pi_{j k} \equiv \frac{\gamma_{k j}}{\gamma_{k}}, \quad k=1, \ldots, N,
$$

where

$$
\gamma_{k j} \equiv \frac{\nu_{k j}^{2}}{\mu_{j}^{2}} \quad \text { and } \gamma_{k} \equiv \sum_{j=1}^{N} \gamma_{k j}, \quad k=1, \ldots, N .
$$

The variance-decomposition proportions are interpreted as follows: $\pi_{j k}$ is the portion of the variance for the $k$ th regression coefficient that is associated with the $j$ th scaled condition index.

Table IV contains the scaled condition indexes and variance-decomposition proportions for the example problem of Fig. 8. The second column contains the scaled condition indexes ranked by size, with the largest shown in the last row. The remaining four columns contain the variancedecomposition proportions for each of the control forces $b_{1}$, $b_{2}, b_{3}$, and $b_{4}$. The next sections explain how to interpret Table IV.

\section{Determine the number of near dependencies}

The method for counting the number of near dependencies involves choosing a threshold $\tilde{\eta}^{*}$ such that when $\tilde{\eta}_{\max }<\tilde{\eta}^{*}$, the columns of $W^{1 / 2} X$ may be said to be free of collinearity. The value suggested by Belsley ${ }^{14}$ is $\tilde{\eta}^{*}=30$, although slightly larger or smaller values could also be argued for. This is the value used for all numerical results in this dissertation. Having chosen a threshold value for $\tilde{\eta}^{*}$, we now have the most basic collinearity diagnostic: whenever $\tilde{\eta}_{\max }<\tilde{\eta}^{*}$, at least one near dependency is present and the regression should not be used without further examination of its collinearity properties. Thus $\tilde{\eta}_{\max }$ should be computed as a routine part of every regression, and displayed along with the $F$-test and $t$-test results.

If more than one scaled condition index exceeds the threshold $\tilde{\eta}^{*}$, then multiple near dependencies are present. The number of near dependencies is determined according to the so-called "progression of 10/30:" the first near dependency corresponds to the first $\tilde{\eta}>30$, the second near depen- 
dency corresponds to $\tilde{\eta}>100$, the third to $\tilde{\eta}>300$, the fourth to $\tilde{\eta}>1000$, and so forth. For example, the sequence of scaled condition indexes $(1,9,33,35)$ indicates only one near dependency, while the sequence $(1,9,33,105,108)$ indicates two near dependencies.

Referring to the first column of Table IV, it is evident that only one scaled condition index exceeds $\tilde{\eta}^{*}$, so we conclude that only one near dependency is present in the example.

\section{Determine actuator involvement}

To determine which actuators are affected by a near dependency, we examine the variance-decomposition proportions $\pi_{j k}$. Having associated a particular $\tilde{\eta}$ with a near dependency, we examine the $\pi_{j k}$ in that row of the table. If two or more actuators have $\pi_{j k}$ in that row larger than some threshold value $\pi^{*}$, then those actuators are involved in the near dependency. The value of $\pi^{*}$ suggested by Belsley ${ }^{14}$ and used in our example is $\pi^{*}=0.5$. In Table IV we examine the last row since that row is associated with a near dependency. Noting that the $\pi_{j k}$ for $b_{1}, b_{2}$, and $b_{3}$ are all well above $\pi^{*}$, we can clearly see that these actuators are involved in the near dependency. (Recall that $b_{1}$ and $b_{2}$ are the ring forces and $b_{4}$ is the ring moment.) The third actuator, with $\pi_{j k}=0.129$, is not involved in the near dependency.

The rules for determining actuator involvement when multiple near dependencies are present are somewhat more complicated and need not be discussed here to illustrate the basic technique. Again, the interested reader is invited to examine Belsley. ${ }^{14}$

\section{Examine auxiliary regressions}

Once we know which actuators are involved in a near dependency, we can regress the individual transfer functions against each other to clarify the relationships between them. For example, we have determined that $b_{1}, b_{2}$, and $b_{4}$ are involved in a near dependency. If we set up a new regression using $b_{4}$ as the disturbance input with $b_{1}$ and $b_{2}$ as control inputs, we can see that the combination of $b_{1}$ and $b_{2}$, if given equal magnitudes and opposite signs, reproduces $b_{4}$ almost exactly (as suspected.) Thus the control input $b_{4}$ could be removed to obtain a more stable regression result.

\section{Determine unaffected actuators}

When an actuator associates most of its variance with small values of $\tilde{\eta}$, we can consider that actuator to be uninvolved with any near dependency. To be more precise, when the total proportion of variance associated with small $\tilde{\eta}^{*}$ is less than $\pi^{*}$, that actuator is uninvolved with any near dependency. In the example problem, the variance of $b_{3}$ is associated almost entirely with the first three small $\tilde{\eta}^{*}$, and therefore we conclude that it is not involved in any near dependency. This is supported by the fact that $b_{3}$ was the least affected by the simulated measurement noise introduced in the example discussed earlier.

\section{SUMMARY}

Multiple linear least-squares regression provides a numerical approach for simulating feedforward active control in the frequency domain. Solving the regression for a given frequency provides the complex control inputs and the amount by which the cost function is reduced. The squared multiple correlation coefficient $\mathbf{R}^{2}$ provides the amount of attenuation possible in the absence of measurement noise. In some cases, particularly where the effects of error sensor measurement noise are a concern, these quantities alone do not adequately characterize the regression. To help model the effects of measurement noise, one may consider the $F$-test, the $t$-test, and prediction intervals. The $F$-test measures the integrity of one the regression as a whole. When the regression fails the $F$-test, as in the example problem whenever the controller performance falls below $2 \mathrm{~dB}$, other regression diagnostics may not be used for that particular frequency. The $t$-test measures the reliability of the estimated control input magnitudes. Prediction intervals combine the information from all the actuators to help describe the sensitivity to noise for the regression as a whole.

Before using regression diagnostics, it is important to carefully examine the statistical properties of the measurement noise present in the proposed error sensors. Strictly speaking, the noise must be normally distributed for the $F$ test, $t$-test, and prediction intervals to be valid. Furthermore, the noise should have constant variance. For some types of sensors, measurement noise may be neither normally distributed nor independent of signal magnitude.

Collinearity problems can produce numerical ill conditioning. Without proper collinearity diagnostics, numerical problems can go undetected and produce results that are very sensitive to measurement noise in the error sensors. Collinearity diagnostics can detect and analyze numerical ill conditioning. Collinearity diagnostics for complex-valued regressions follow directly from their real-valued counterparts, which are developed in considerable detail in the statistics literature. The primary collinearity diagnostic is the scaled condition number. Even when no other collinearity diagnostics are performed, the analyst may compute the scaled condition number for each regression and compare it to the threshold value in a somewhat mechanical fashion. If the scaled condition number exceeds the threshold, the regression should be discarded because of collinearity. More detailed diagnostics are also available for determining the number of near dependencies and which control forces are involved.

\section{ACKNOWLEDGMENTS}

The authors gratefully acknowledge financial support from Carderock Division, Naval Surface Warfare Center and from the Office of Naval Research (Grant No. ONRN00014-88-K-0721). The authors also thank Dr. Alan Miller (CSIRO) and Dr. Kenneth White (University of British Columbia) for generously providing advice concerning regression and general statistical considerations. Felix Rosenthal provided helpful input concerning singular-value decomposition. 
${ }^{1}$ P. A. Nelson and S. J. Elliott, The Active Control of Sound (Academic, London, 1992)

${ }^{2}$ P. A. Nelson, A. R. D. Curtis, S. J. Elliott, and A. J. Bullmore, "The active minimization of harmonic enclosed sound fields, Part I: Theory," J. Sound Vib. 117, 1-13 (1987).

${ }^{3}$ A. J. Bullmore, P. A. Nelson, A. R. D. Curtis, and S. J. Elliott, "The active minimization of harmonic enclosed sound fields, Part II: A computer simulation," J. Sound Vib. 117, 15-33 (1987).

${ }^{4}$ C. G. Mollo and R. J. Bernhard, "Generalized method of predicting optimal performance of active noise controllers," AIAA J. 27, 1473-78 (1989). Note: author's name misspelled as "Molo."

${ }^{5}$ L. Song, G. H. Koopmann, and J. B. Fahnline, "Active control of the acoustic radiation of a vibrating structure using a superposition formulation," J. Acoust. Soc. Am. 89, 2786-2792 (1991).

${ }^{6} \mathrm{~L}$. Ljung, System Identification: Theory for the User (Prentice-Hall, Englewood Cliffs, NJ, 1987).

${ }^{7}$ B. Wahlberg and L. Ljung, "Hard frequency-domain model error bounds from least-squares like identification techniques," IEEE Trans. Autom. Control 37, 900-912 (1992).

${ }^{8}$ S. D. Snyder, "A fundamental study of active noise control system design," unpublished Dissertation, Department of Mechanical Engineering, University of Adelaide, 1991.

${ }^{9}$ S. D. Snyder and C. H. Hansen, "Using multiple regression to optimize active noise control system design," J. Sound Vib. 148, 537-542 (1990).

${ }^{10}$ K. S. Miller, "Complex Gaussian processes," SLAM Rev. 11, 544-567 (1969).

${ }^{11}$ K. S. Miller, "Complex linear least squares," SIAM Rev. 15, 707-726 (1975).

${ }^{12} \mathrm{~F}$. Rosenthal, "A new technique for the active cancellation of wide-band noise using multiple sensors," Proceedings of the Second International Congress on Recent Developments in Air- and Structure-borne Sound and Vibration, March 1992 (unpublished), Vol. 1, pp. 337-344.

${ }^{13}$ D. A. Belsley, E. Kuh, and R. E. Welsch, Regression Diagnostics: Identifying Inftuential Data and Sources of Collinearity (Wiley, New York, 1980).

${ }^{14}$ D. A. Belsley, Conditioning Diagnostics: Collinearity and Weak Data in Regression (Wiley, New York, 1991).

${ }^{15}$ C. E. Ruckman, "Method and example application for least squares regression of complex-valued variables," Appl. Statistics (submitted 1994).

${ }^{16} \mathrm{C}$. E. Ruckman, "A regression-based approach for simulating active noise control in the frequency domain, with application to fluid-structure inter- action problems," Doctoral dissertation, Virginia Polytechnic Institute and State University, May 1994.

${ }^{17} \mathrm{C}$. R. Fuller and J. D. Jones, "Experiments on reduction of propellerinduced interior noise by active control of cylinder vibration," J. Sound Vibr. 112, 389-395 (1987).

${ }^{18} \mathrm{H}$. C. Lester and C. R. Fuller, "Active control of propeller induced noise fields inside a flexible cylinder," AIAA J. 28, 1374-1380 (1989).

${ }^{19}$ R. L. Clark and C. R. Fuller, "Control of sound radiation with adaptive structures," J. Intelligent Mater. Syst. Struct. 2, 431-45 (1991).

${ }^{20}$ C. R. Fuller and R. A. Burdisso, "A wavenumber domain approach to the active control of structure borne sound," J. Sound Vib. 148, 355-360 (1991).

${ }^{21}$ C. R. Fuller, C. H. Hansen, and S. D. Snyder, "Active control of sound radiation from vibrating rectangular panel by sound sources and vibration inputs: An experimental comparison," J. Sound Vib. 145, 195-215 (1991).

${ }^{22} \mathrm{C}$. R. Fuller, "Active control of sound transmission/radiation from elastic plates by vibration inputs: I. Analysis," J. Sound Vib. 136, 1-15 (1990).

${ }^{23}$ Y. Gu and C. R. Fuller, "Active control of sound radiation due to subsonic wave scattering from discontinuities on fluid-loaded plates. I: Far-field pressure," J. Acoust. Soc. Am. 90, 2020-2026 (1991).

${ }^{24} \mathrm{C}$. Guigou and C. R. Fuller, "Active control of edge sound radiation from a semi-infinite elastic beam with a clamped edge: Influence of bending near-field waves," J. Acoust. Soc. Am. 93, 2716-25 (1992).

${ }^{25}$ C. E. Ruckman and C. R. Fuller, "Numerical simulation of active structural-acoustic control for a fluid-loaded, spherical shell: Formulation and validation," J. Acoust. Soc. Am. 96, 2817-2825 (1994).

${ }^{26}$ A. M. Neville and J. B. Kennedy, Basic Statistical Methods for Engineers and Scientists (International Textbook, Scranton, PA, 1964).

${ }^{27}$ N. R. Draper and H. Smith, Applied Regression Analysis (Wiley, New York, 1966).

${ }^{28}$ C. L. Lawson and R. J. Hanson, Solving Least Squares Problems (Prentice-Hall, Englewood Cliffs, NJ, 1974).

${ }^{29}$ R. J. Carroll and D. Ruppert, Transformation and Weighting in Regression (Chapman and Hall, New York, 1988).

${ }^{30}$ M. C. Junger and D. Feit, Sound, Structures, and Their Interaction (MIT, Cambridge, 1986), 2nd ed.

${ }^{31}$ G. C. Everstine and A. J. Quezon, "User's guide to the coupled NASTRAN/Helmholtz equation capability (NASHUA) for acoustic radiation and scattering," CDNSWC-SD-92-17, Carderock Division, NSWC, Bethesda, MD, 1992. 